\title{
PESQUISA OPERACIONAL: UM ESTUDO SOBRE A UTILIZAÇÃO DA FERRAMENTA SOLVER NA TOMADA DE DECISÃO DE PROBLEMAS DE PRODUÇÃO AGRÍCOLA
}

\author{
Douglas Fernandes, Flavio Alberto Oliva, Marcus Ayrton Rocha de Lima
}

Universidade do Oeste Paulista - UNOESTE, Faculdade de Engenharia de Produção, Presidente Prudente, SP. E-mail: marcus.lima5@fatec.sp.gov.br.

\section{RESUMO}

O cenário de trabalho empírico dos pequenos produtores rurais brasileiros, em nada foge à realidade das empresas e dos empreendedores em nosso país em todas as outras áreas. As tomadas de decisão, ações extremamente importantes e necessárias no dia a dia dos negócios da grande maioria dos pequenos produtores e produtores familiares, são feitas sem base de conhecimento ou metodologias e recursos aplicáveis. Isso torna estes empreendimentos vulneráveis às muitas e frequentes variáveis. O quê plantar, o quanto plantar, seus custos e lucratividade, são algumas das questões que margeiam a mente dos produtores. A informação, base da tomada de decisão, é precária ou quase nula. Para realizar este trabalho, foi feito um levantamento bibliográfico e a criação de um problema hipotético envolvendo um pequeno produtor rural de olerícolas, a partir do qual, foi estruturado um modelo matemático usando conhecimentos de Pesquisa Operacional (PO), modelagem em Solver no Microsoft Excel com método Simplex e conceitos de negócios. Assim, duas simulações foram geradas a partir do modelo estabelecido, uma de alcance de ponto de equilíbrio e outra de maximização de recursos para obtenção da maior lucratividade. O modelo formatado com o objetivo de resolver o problema hipotético aqui relatado, proporcionou, de maneira simples e rápida gerar as simulações supracitadas, facilitando sobremaneira e fornecendo alternativas para a tomada de decisão do grupo aqui estudado. Outrossim, foi verificado que há a possibilidade de utilizar ferramentas e metodologias aplicáveis como suporte a esse público carente de forma simples, barata e rápida.

Palavras-chave: Olericultura, Solver, Simplex, Decisão, Produtores

\section{OPERATIONAL RESEARCH: A STUDY ON THE USE OF THE TOOL SOLVER IN THE DECISION MAKING OF AGRICULTURAL PRODUCTION PROBLEMS}

\begin{abstract}
The empirical work scenario of the small Brazilian rural producers, in no way escapes the reality of companies and entrepreneurs in our country in all other areas. Decision-making, extremely important and necessary actions in the day-to-day business of the vast majority of small producers and family producers, are made without a knowledge base or applicable methodologies and resources. This makes these ventures vulnerable to many, many variables. What to plant, how much to plant, its costs and profitability, are some of the issues that border the producers' minds. Information, the basis of decision-making, is precarious or almost nil. In order to carry out this work, a bibliographical survey and the creation of a hypothetical problem involving a small rural producer of vegetables, from which a mathematical model was structured using Operational Research knowledge, modeling in Solver in Microsoft Excel With Simplex method and business concepts. Thus, two simulations were generated from the established model, one of reach of equilibrium point and one of maximization of resources to obtain the highest profitability. The model formatted with the objective of solving the hypothetical problem reported here, provided, in a simple and fast way to generate the simulations mentioned above, facilitating greatly and
\end{abstract}


providing alternatives for the decision making of the group studied here. Also, it was verified that there is the possibility of using applicable tools and methodologies to support this needy public in a simple, cheap and fast way.

Keywords: Olericulture, Solver, Simplex, Decision, Producers 


\section{INTRODUÇÃO}

A tônica do trabalho do gestor é a tomada de decisão, este momento considerado bastante crítico dentre suas funções é altamente importante para os negócios, pois a decisão errada pode acarretar consequências sérias às empresas. Por esta razão é necessário munir-se de informações para ajudá-lo nessa tarefa árdua e difícil.

De acordo com Freitas et al. (1997, p. 51), "a atividade de tomar decisões é crucial para as organizações. Esta atividade acontece todo o tempo, em todos os níveis, e influencia diretamente o desempenho da organização".

Pesquisas em administração rural demonstram que o produtor dá início ao processo decisório, que é intrínseco e particular às suas individualidades, quanto aos recursos disponíveis em seu meio e as limitações referentes às restrições e finaliza o processo nele mesmo (CARRIERI, 1992).

A tomada de decisão pode ser conceituada, de maneira simples, como escolha entre alternativas com o objetivo de resolver problemas e aproveitar oportunidades. A base da tomada de decisão é a informação.

Uma das metodologias disponíveis aos gestores é a Pesquisa Operacional (PO), área do conhecimento que pode proporcionar a geração de informações e simulações. Este conceito que surgiu durante a Segunda Guerra Mundial, inicialmente para resolver problemas logísticos e de estratégia e tática militares de grandes dimensões e complexidade. Para apoiar comandos militares e proporcionar tomada de decisões e melhoria de soluções. Foram reunidos grupos multidisciplinares de matemáticos, físicos, engenheiros e cientistas sociais, que também se utilizaram da Teoria dos Jogos, trazendo para situações reais, simulações aplicáveis, inicialmente a situações de guerra.

Tal experiência foi tão interessante, de resultados altamente positivos, que rapidamente esse conhecimento foi absorvido, aperfeiçoado e aplicado aos problemas empresariais, tal qual conhecemos hoje como Pesquisa Operacional.

São cálculos e modelos matemáticos para resolver problemas do dia a dia dos negócios, como por exemplo: logística, produção, vendas, marketing, finanças entre outras.

Segundo a SOBRAPO (Sociedade Brasileira de Pesquisa Operacional), "Pesquisa Operacional ( $\mathrm{PO}$ ) é a área de conhecimento que estuda, desenvolve e aplica métodos analíticos avançados para auxiliar na tomada de melhores decisões nas mais diversas áreas de atuação humana".

Assim como qualquer empresa, independente do ramo de atividade, os pequenos produtores rurais, agricultores familiares, carecem de ferramentas para auxiliá-los em suas decisões que, normalmente são empíricas.

O objetivo deste trabalho é demonstrar que, com cálculos simples, utilização de uma metodologia aplicável e ferramentas disponíveis em planilha eletrônica, é possível gerar um modelo matemático para ajudar esses pequenos produtores rurais em suas decisões. Aqui, especificamente, decisões relativas a: o que produzir e em quê quantidades, já que as restrições são fatores que dificultam suas decisões, além de suas limitações de cunho pessoal.

\section{METODOLOGIA}

Em atendimento aos objetivos propostos neste trabalho, primeiramente lugar foram realizadas pesquisas bibliográficas com relação ao tema; em um segundo momento foi gerado um problema hipotético de produção olerícola em uma pequena área rural de $400 \mathrm{~m}^{2}$.

O termo Olericultura é derivado do latim olus hortaliça e colere cultivar e, portanto, é utilizado para designar o cultivo de certas plantas de consistência herbácea, geralmente de ciclo curto e tratos culturais intensivos, cujas partes comestíveis são diretamente utilizadas na alimentação humana, sem exigir industrialização prévia. As hortaliças também são denominadas 
por cultura olerácea e são popularmente conhecidas como verduras e legumes. No Dicionário Aurélio da Língua Portuguesa (Brasil) olericultura é definida como: "cultivo de legumes";

Para analisar os dados aqui apresentados, foi empregado o método Simplex para a resolução de problemas e utilizada a ferramenta Solver para modelar o problema. O Solver está presente em planilhas eletrônicas Microsoft Excel, portanto, de fácil acesso e baixo investimento. Variáveis Hipotéticas:

1) Três tipos de olerícolas para plantio: Alface, almeirão e rúcula;

2) Uma área plantável de $400 \mathrm{~m}^{2}$;

3) Espaçamento em centímetros entre as plantas e a capacidade total de plantio, conforme a tabela abaixo:

Tabela 1. Tipos de olerícolas e espaçamento entre plantas

\begin{tabular}{|l|r|r|}
\hline CULTURA & ESPAÇAMENTO & CAPAC. TOTAL \\
\hline ALFACE & 30 & $4.444,44$ \\
\hline ALMEIRÃO & 27 & $5.486,97$ \\
\hline RÚCULA & 25 & $6.400,00$ \\
\hline
\end{tabular}

Fonte: Os autores

4) Capital de Giro disponível de $\mathrm{R} \$ 10.000,00$

5) Demanda mensal de:

Tabela 2. Demanda mensal

DEMANDA MENSAL - PÉS
\begin{tabular}{|l|r|}
\hline \multicolumn{1}{|c|}{ CULTURA } & \multicolumn{1}{c|}{ QTIDADE } \\
\hline ALFACE & 10.000 \\
\hline ALMEIRÃO & 7.000 \\
\hline RÚCULA & 4.000 \\
\hline
\end{tabular}

Fonte: Os autores

6) Preço e Custo variável unitário:

Tabela 3. Preços unitários e custos variáveis unitários

\begin{tabular}{|l|ll|ll|}
\hline CULTURA & \multicolumn{2}{|c|}{$P U$} & \multicolumn{2}{c|}{ CVU } \\
\hline ALFACE & $\mathrm{R} \$$ & 2,50 & $\mathrm{R} \$$ & 1,80 \\
\hline ALMEIRÃO & $\mathrm{R} \$$ & 2,70 & $\mathrm{R} \$$ & 1,90 \\
\hline RÚCULA & $\mathrm{R} \$$ & 3,00 & $\mathrm{R} \$$ & 2,00 \\
\hline
\end{tabular}

Fonte: Os autores

PU: Preço Unitário

CVU: Custo Variável Unitário 
7) Custo Fixo de $R \$ 3.500,00$ mensais.

\section{DISCUSSÃO}

A área agricultável, o capital de giro, a demanda, o preço e o custo são restrições hipotéticas ao negócio. O modelo deverá apresentar as melhores situações de produção (quantidade) que o empresário poderá adotar como decisão para atingir um determinado resultado ou maximizá-lo.

Como primeiro passo, foi feita uma simulação para que o modelo apresentasse quais condições deveriam ser cumpridas a se chegar ao Ponto de Equilíbrio (Ponto de faturamento em que os custos variáveis e fixos sejam cobertos pela receita), ou seja, quais quantidades plantar de cada cultura para o atendimento das restrições e alcance do resultado desejado.

Tabela 4. Demonstração do resultado gerencial no ponto de equilíbrio e as margens de contribuição

\begin{tabular}{|c|c|c|c|c|c|c|c|c|}
\hline CULTURA & QTD PÉS & \multicolumn{2}{|c|}{$\mathrm{PU}$} & CVU & \multicolumn{2}{|c|}{$\mathrm{MCU}$} & \multicolumn{2}{|r|}{ MCT } \\
\hline ALFACE & - & $\mathrm{R} \$$ & 2,50 & $\mathrm{R} \$ 1,80$ & $\mathrm{RS}$ & 0,70 & $\mathrm{RS}$ & - \\
\hline ALMEIRÃO & - & $\mathrm{RS}$ & 2,70 & $\mathrm{R} \$ \mathbf{1 , 9 0}$ & $\mathrm{R} \$$ & 0,80 & $\mathrm{RS}$ & - \\
\hline RÚCULA & 3.500 & $\mathrm{R} \$$ & 3,00 & $R \$ 2,00$ & $\mathrm{R} \$$ & 1,00 & $\mathrm{R} \$$ & $3.500,00$ \\
\hline TOTAL & 3.500 & & & & SON & MCT & & $3.500,00$ \\
\hline & & & & & $(-)$ & IXO & & $3.500,00$ \\
\hline & & & & & RESI & TADO & & - \\
\hline
\end{tabular}

Fonte: Os autores

MCU: Margem de Contribuição Unitária (Resultado de Receitas menos os gastos variáveis).

MCT: Margem de Contribuição Total (Multiplicação da quantidade pela MCU).

Nesta simulação, a solução apresentada pelo modelo foi plantar somente Rúcula (3.500 pés), pois o Capital de Giro a ser utilizado seria de $\mathrm{R} \$ 7.000,00$ (3.500 X 2,00).

Com essa primeira simulação a capacidade instalada que é de $400 \mathrm{~m}^{2}$ foi aproveitada em $54,69 \%$, conforme observado na tabela abaixo:

Tabela 5. Utilização da capacidade produtiva no ponto de equilíbrio

\begin{tabular}{|c|c|c|c|c|}
\hline PROD & CAP. $M^{2}$ & QTD & ÁREA USADA M ${ }^{2}$ & $\%$ USO \\
\hline ALFACE & 11,11 & - & - & $0,00 \%$ \\
\hline ALMEIRÃO & 13,72 & - & - & $0,00 \%$ \\
\hline RÚCULA & 16,00 & 3.500 & 219 & $54,69 \%$ \\
\hline & & TOTAL & 219 & $54,69 \%$ \\
\hline
\end{tabular}

Fonte: Os autores

A Segunda Simulação foi realizada com o objetivo de maximização do resultado, ou seja, qual quantidade máxima que poderia ser plantada, dadas as restrições, para que o empresário alcançasse seu maior lucro. É o que podemos observar na tabela abaixo: 
Tabela 6. Demonstração do resultado gerencial na maximização do resultado e as margens de contribuição

\begin{tabular}{|c|c|cr|r|rr|rr|}
\hline CULTURA & QTD PÉS & \multicolumn{2}{|c|}{ PU } & \multicolumn{2}{c|}{ CVU } & \multicolumn{2}{c|}{ MCU } & \multicolumn{2}{c|}{ MCT } \\
\hline ALFACE & - & R\$ & 2,50 & R\$ 1,80 & $\mathrm{R} \$$ & 0,70 & $\mathrm{R} \$$ & - \\
\hline ALMEIRÃO & 1.053 & $\mathrm{R} \$$ & 2,70 & $\mathrm{R} \$ 1,90$ & $\mathrm{R} \$$ & 0,80 & $\mathrm{R} \$$ & 842,11 \\
\hline RÚCULA & 4.000 & $\mathrm{R} \$$ & 3,00 & $\mathrm{R} \$ 2,00$ & $\mathrm{R} \$$ & 1,00 & $\mathrm{R} \$$ & $4.000,00$ \\
\hline
\end{tabular}

\begin{tabular}{|l|r|}
\hline TOTAL & 5.053 \\
\hline
\end{tabular}

\begin{tabular}{|l|r|}
\hline SOMA MCT & $4.842,11$ \\
\hline ( - ) CFIXO & $3.500,00$ \\
\hline RESULTADO & $1.342,11$ \\
\hline
\end{tabular}

Fonte: Os autores

O resultado máximo, dadas as variáveis e restrições impostas à tomada de decisão é de R\$ 1.342,11. O modelo sugere ao tomador de decisão que plante somente almeirão, com 1.053 pés e rúcula com 4.000 pés. Assim, esta simulação demonstra abaixo a utilização da capacidade instalada, que chegou a $81,68 \%$ do total de $400 \mathrm{~m}^{2}$ e uso total do capital de giro disponível:

Tabela 7. Utilização da capacidade produtiva - Maximização

\begin{tabular}{|c|c|c|c|c|}
\hline CULTURA & CAP. $M^{2}$ & QTD & ÁREA USADA M ${ }^{2}$ & $\%$ USO \\
\hline ALFACE & 11,11 & - & - & $0,00 \%$ \\
\hline ALMEIRÃO & 13,72 & 1.053 & 77 & $19,18 \%$ \\
\hline RÚCULA & 16,00 & 4.000 & 250 & $62,50 \%$ \\
\hline & & TOTAL & 327 & $81,68 \%$ \\
\hline
\end{tabular}

As simulações foram feitas utilizando a ferramenta SOLVER, presente em qualquer planilha do Microsoft Excel com o método de algoritmo Simplex, que é um método de otimização para resolver equações lineares (programação linear). O modelo foi formatado em uma planilha Microsoft Excel.

\section{CONCLUSÃO}

O estudo foi realizado a partir de um cenário fictício de variáveis que estão presentes diariamente na vida do pequeno produtor rural. Suas decisões, que são feitas de maneira empírica, sem base em cálculos de custo e sem qualquer tipo de simulação o tornam altamente vulnerável às condições de mercado. Essa condição de instabilidade pode ser diminuída com a utilização de recursos e áreas do conhecimento apropriados, como é o caso da P.O. O estudo permitiu-nos concluir que, apesar das dificuldades presentes nos processos de tomada de decisão dos pequenos produtores rurais, das variáveis que influenciam o seu negócio, algumas questões podem ser respondidas de maneira rápida, eficiente e eficaz com o uso de ferramentas e metodologias aplicáveis; recursos estes, e ao alcance do produtor de maneira a auxiliá-lo com grandes benefícios na árdua tarefa de tomar decisões acertadas. Conferindo aos pequenos produtores rurais a possibilidade de realizar simulações antes de partir para a ação.

\section{REFERÊNCIAS BIBLIOGRÁFICAS}

CARRIERI, Alexandre de Pádua. A racionalidade administrativa: os sistemas de produção e o processo de decisão-ação em unidades de produção rural. Lavras. 208 p. Dissertação (Mestrado) Escola Superior de Agricultura de Lavras, Universidade Federal - Minas Gerais, 1922. 
FREITAS, H. et al. Informação e decisão: sistemas de apoio e seu impacto. Porto Alegre: Ortiz, 1997. $214 \mathrm{p}$.

FERREIRA, Aurélio B. de Hollanda. Novo Dicionário da Língua Portuguesa. 2. ed. Rio de Janeiro: Nova Fronteira, 1986. 1838 p.

SOBRAPO - Sociedade Brasileira de Pesquisa Operacional. Disponível em: http://www.sobrapo.org.br/. Acesso em: 12 de jun.2017. 